Revista de Derecho

\title{
El principio de universalidad desde una perspectiva legal y efectiva en la seguridad social ecuatoriana
}

\section{The principle of universality from a legal and effective perspective in ecuadorian social security}

\author{
María Dolores Núñez Ávila \\ Investigadora independiente \\ Ecuador \\ dolonuneza@gmail.com; madonua@alumni.uv.es \\ ORCID: 0000-0002-4705-8002
}

\author{
María Lorena Rivadeneira Vásquez \\ Investigadora independiente \\ Ecuador \\ lorerivas90@hotmail.com \\ ORCID: 0000-0001-9932-4790
}

DOI: https://doi.org/10.32719/26312484.2021.35.7

Fecha de recepción: 30 de junio de 2020

Fecha de aceptación: 22 de septiembre de 2020

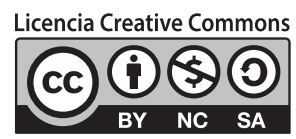




\section{RESUMEN}

Los principios que rigen la seguridad social se encuentran reconocidos por la Constitución de 2008, entre los cuales encontramos el principio de universalidad. Estos principios constituyen una herramienta de evaluación y hermenéutica a los distintos sistemas de seguridad social. En este sentido, el principio de universalidad representa un instrumento de igualdad social que rompe con el esquema de clase con el que fue creado la seguridad social. En Ecuador a raíz de la Constitución de 2008 encontramos la extensión de la cobertura carácter universal hacia los trabajadores no remunerados del hogar. Por este motivo el objetivo de este artículo se centra en analizar el principio de universalidad desde un enfoque legal y efectivo junto con el sistema de seguridad social contributivo y no contributivo aplicado en Ecuador e identificar la operatividad del Instituto Ecuatoriano de Seguridad Social y del Banco del Instituto Ecuatoriano de Seguridad Social de conformidad con el principio de universalidad efectiva. Para esto se ha realizado un análisis deductivo-inductivo conceptual, doctrinal, legal, jurisprudencial; que ha permitido detectar ciertos elementos que limitan a la universalidad efectiva en el acceso a la seguridad social, como también aquellos vacíos estructurales que desamparan a distintos sectores de la población.

PALABRAS CLAVE: seguridad social, universalidad, IESS, BIESS, contribución, Ecuador.

\section{ABSTRACT}

The Constitution of Ecuador recognizes the principles of social security. These principles constitute an evaluation and hermeneutic tool for the different social security systems. In these principles, we find the universality; the principle of universality is an instrument of social equality, and it broke the class scheme in which social security. In Ecuador, since 2008, universal coverage of social security has been extended to unpaid household workers. The man object analyzes the principle of universality from a legal approach, the contributory and non-contributory social security system applied in Ecuador, and the analysis of the functioning of the Ecuadorian Institute of Social Security and the Bank of the Ecuadorian Institute of Social Security. For this, a deductive-inductive conceptual, doctrinal, legal, jurisprudential, and pragmatic analysis has allowed the detection of specific elements limiting access to social security and structural gaps forsaking sectors of the population, specifically those covered by the non-contributory model.

KEYwORDs: Social Security, Universality, IESS, BIESS, Contribution, Ecuador. 


\section{INTRODUCCIÓN}

$\mathrm{E}$ sistema ecuatoriano de seguridad social es el encargado de brindar una vida digna a cada individuo de la sociedad; es decir, bienestar social desde el nacimiento hasta su muerte a través del Instituto Ecuatoriano de Seguridad Social (IESS) y del Banco del Instituto Ecuatoriano de Seguridad Social (BIESS); lo que implica una adecuada gestión, administración y control de tres regímenes que facilitan el acceso a diferentes tipos de beneficios sociales. Existe una disparidad en cuanto a lo que universalidad y cobertura se refiere, ya que hay personas que han sido excluidas de los regímenes antes mencionados o han recibido un trato desfavorable al acceder a la seguridad social, así como irresponsabilidad en el manejo de fondos por parte de los funcionarios que lideran las instituciones, políticas que no se cumplen y la deuda estatal, que han producido una crisis económica y social de gran envergadura en las entidades, convirtiendo a la seguridad social del Ecuador en un servicio público deficiente.

\section{PRINCIPIO DE UNIVERSALIDAD EN LA SEGURIDAD SOCIAL: ANÁLISIS DE LA UNIVERSALIDAD LEGAL Y EFECTIVA}

La Organización Internacional del Trabajo señala que debido a la complejidad de la materia es imposible dar una definición de carácter universal; considera que la seguridad social:

abarca todas las medias relacionadas con las prestaciones, en efectivo o en especie, encaminadas a garantizar: la falta de ingresos laborales debido a enfermedad, discapacidad, maternidad, accidentes de trabajo, desempleo, vejez o muerte de un miembro de la familia; falta de acceso o precios excesivos en el acceso a la asistencia médica; apoyo familiar insuficiente, en particular para los hijos y adultos a cargo; pobreza generalizada y exclusión social. ${ }^{1}$

Para Betranou, "El término puede incluir al seguro social, a la asistencia social, a los regímenes de prestaciones mutuales, a los fondos de previsión y a los otros regímenes especiales". ${ }^{2}$ La seguridad social no se ve restringida únicamente al acceso al derecho de la salud, sino que busca la estabilidad cotidiana mediante sistemas de pensiones, rubros de derecho habiencia y salud. ${ }^{3}$

1. OIT, Informe VI: Seguridad Social para justicia social y una globalización equitativa (Ginebra: OIT, 2011 ), 8.

2. Fabio Bertranou, "Restrictions, problems and dilemmas of social protection in Latin America: Facing the challenges from ageing and income security", Well-being and Social Policy 1, n. ${ }^{\circ} 1$ (2005): 33-54.

3. Roberto Ham, "De la solidaridad intergeneracional a la privatización de las pensiones", Carta Demográfica sobre México (Ciudad de México: Demos, 1996), 36-7. 
Existe una serie de principios que establece la guía para los distintos componentes del sistema de seguridad social; para Calvo León, estos principios son una herramienta de orientación, hermenéutica, evaluación de los modelos de seguridad social, que permiten identificar los ajustes necesarios para garantizarlo, estableciendo así parámetros para enjuiciar los grados de desarrollo; ${ }^{4}$ además, se interrelacionan entre sí y son el principio de universalidad, solidaridad, unidad, igualdad, evolución progresiva de los beneficios de la seguridad social, concordancia de la seguridad social con la realidad económica, participación social, integralidad, inmediatez, subsidiaridad del Estado, asignación preferente de recursos. ${ }^{5}$ El objeto de estudio del presente artículo se centra únicamente en el principio de universalidad.

La importancia del principio de universalidad como fundamento en el acceso a la seguridad social es indiscutible; de acuerdo con la OIT este principio representa una herramienta de igualdad social ${ }^{6}$ que se opone al carácter clasista obrero y exclusivo del cual tuvo origen y busca la protección del ser humano, dentro de una colectividad social indistintamente de la clase a la que pertenezca o de su actividad laboral. ${ }^{7}$ Para Juan Bernaldo De Quiros, el derecho de acceso al seguro social no implica una misión privativa de clase, sino un concepto integral de protección a la colectividad. ${ }^{8}$

Para Melik Ózdem, la puesta en práctica del derecho a la seguridad social depende de la existencia del buen funcionamiento de un sistema de seguridad social; ${ }^{9}$ Etchichury considera que, dependiendo de la noción de universalidad que tenga un país dentro de su marco normativo, el diseño de políticas públicas tendrá un determinado perfil; si los derechos pueden ser ejercidos libremente por todas las personas que se encuentran dentro de la jurisdicción de un Estado, las políticas públicas tendrán una mayor extensión a diferencia de aquellos Estados que tienen una noción restringida del alcance de los derechos. ${ }^{10}$

Para la OIT, la universalidad puede ser analizada desde un enfoque legal como efectivo y se relacionan las condiciones establecidas en la legislación para su acceso;

4. Jorge Iván Calvo León, "Principios de seguridad social", Revista Jurídica de Seguridad Social, n. ${ }^{\circ} 8$ (1998): 36-40, https://bit.ly/3bIxg9u.

5. Ibíd.

6. OIT, Panorama temático laboral. Presente y futuro de la protección social en América Latina y el Caribe (Ginebra: OIT, 2018), 81-3, https://bit.ly/3fLMqMt.

7. Calvo León, "Principios de seguridad social", 36.

8. Juan Bernaldo De Quiroz, “Concepto y fundamento”, El Seguro Social en Iberoamérica 44 (1945): 11-6, doi: $10.2307 /$ j.ctvckq2zk.4.

9. Melik Özden, El derecho a la Seguridad. Ginebra, 9.

10. Horacio Javier Etchichury, "Universalidad y derechos sociales: para una revisión constitucional de las políticas sociales en Argentina”, Revista Estudios Socio-Jurídicos, n. ${ }^{\circ}$ (2019): 327-54, doi: http://dx.doi. org/10.12804/revistas.urosario.edu.co/sociojuridicos/a.6501. 
en el caso de la universalidad efectiva en ciertos casos se ve limitada por un esquema contributivo legal, que determina el acceso a la seguridad social mediante el cumplimiento de distintos requisitos formales establecidos por el régimen jurídico interno de cada Estado. ${ }^{11}$

Por otra parte, puede existir el principio de universalidad de naturaleza legal, pero no llega a constituirse como un principio de universalidad efectiva, por la inexistencia de una cobertura universal efectiva. ${ }^{12}$ Esto se debe a que la universalidad efectiva busca una cobertura global, es decir una cobertura efectiva, para lo cual, dentro de un régimen contributivo, se busca mediante un régimen no contributivo extender la cobertura hacia ciertos sectores vulnerables. Es aquí cuando se concibe que la universalidad es una herramienta de reivindicación social.

Existe doctrina que considera que el sistema de seguro social surge de la relación de dependencia laboral ${ }^{13}$ y excluye a aquellos ciudadanos que no cuentan con estabilidad laboral o se encuentran desempleados; sin duda este es uno de los principales retos que enfrenta la universalidad efectiva. ${ }^{14}$

Para el Comité de Derechos Económicos, Sociales y Culturales de la ONU (Codesc) la accesibilidad del sistema de seguridad social obedece a los criterios de cobertura como derecho humano universal, la admisibilidad sin condición particular, en el que las cotizaciones no deberían ser prohibitivas, permitiendo el acceso físico y la participación e información, además considera que es importante identificar a la seguridad social como un bien social y no como un instrumento de política económica o financiera. ${ }^{15}$

La consecución de un sistema de seguridad social en virtud del principio de universalidad efectiva representa desafíos importantes relacionados con la cobertura, equidad, fragmentación, sostenibilidad, previsibilidad y litigiosidad. ${ }^{16}$ Ruiz Moreno considera que la problemática del derecho a la seguridad social deviene de dos vertientes: a) de la justificación sobre los altos costes que representa la seguridad social universal para el Estado y la falta de recursos para llevarlos a cabo, propio del debate que generan los derechos sociales en la práctica; y b) la naturaleza pentagonal (multidisciplinar) de la materia y que es difícil dar una solución óptima si no se concibe la

11. OIT, Panorama temático laboral. Presente y futuro de la protección social, 81-2. https://bit.ly/3fLMqMt.

12. Ibíd.

13. Marco Proaño Maya, Seguridad social y sociedad democrática (Quito: Editorial Americana, 2014), 89.

14. OIT, Panorama temático laboral, presente y futuro de la protección social en América, 82.

15. CODESC, Observación General, $n .^{\circ} 19$ (Ginebra: ONU, 2007), citado en Melik Özden, El derecho a la Seguridad.

16. ONU, CEPAL, OIT, "Encrucijadas en la seguridad social argentina: reformas, cobertura y desafíos para el sistema de pensiones" (Buenos Aires: ONU / CEPAL / OIT, 2011): 141, https://bit.ly/3bOmhLy. 
seguridad social como un todo que involucra las ramas del derecho laboral, derecho tributario, derecho económico y derecho financiero, desde un punto de vista doctrinario, pragmático y legal. ${ }^{17}$ Esto se debe a la institucionalización de la desigualdad que, a pesar de los intentos de eliminar este fenómeno, se reitera con facilidad en la región latinoamericana, ${ }^{18}$ generando pobreza e inseguridad y debilitando al capital humano. ${ }^{19}$

Rey del Castillo analiza el principio de universalidad efectiva en la seguridad social y considera que la universalidad legal en ocasiones genera mayor confusión; la exclusión de ciertos grupos sociales en el acceso al seguro social pone en cuestión la equidad de la prestación. ${ }^{20}$ Es importante para el alcance de la universalidad efectiva el reconocimiento normativo por parte de los Estados de prestaciones no contributivas para construir un sistema de cobertura universal, para que de esta manera se constituya en una herramienta de redistribución de la riqueza e igualdad, en el que los Estados se comprometan a prevenir riesgos y exista una cobertura universal hacia toda la colectividad. ${ }^{21}$

\section{EL PRINCIPIO DE UNIVERSALIDAD EN EL RÉGIMEN JURÍDICO INTERNACIONAL Y REGIONAL}

Ecuador ha asumido distintos compromisos internacionales de naturaleza vinculante (hard law) y no vinculante (soft law). Los instrumentos que se encuentran dentro del grupo conocido como hard law poseen fuerza coercitiva, a diferencia de los instrumentos que se agrupan dentro de soft law; esto significa que estos últimos carecen una fuerza coactiva y se configura en una obligación de carácter moral. ${ }^{22}$

Ecuador es un Estado parte de la Declaración Universal de Derechos Humanos y el Pacto Internacional de Derechos Económicos Sociales y Culturales (PIDESC). ${ }^{23}$ Estos

17. Ángel Guillermo Ruiz Moreno, "Retos y desafíos de la seguridad social latinoamericana: entre la realidad y la utopía", Revista Latinoamericana de Derecho Social, n. ${ }^{\circ} 12$ (2011): 130, https://bit.ly/3m2u3Gp.

18. Boaventura de Sousa Santos, Sociología jurídica crítica para un sentido común en el Derecho (Bogotá: Trotta, 2009), 49.

19. Elizabeth Tinoco, "El desafío de la seguridad social en América Latina", Organización Internacional del Trabajo, 6 de febrero de 2015, https://bit.ly/3fLPuIs.

20. Javier Rey del Castillo, "Seguridad social y universalidad: ¿son compatibles?, Revista de Administración Sanitaria Siglo XXI, n. 3 (2004): 409-31, https://bit.ly/2ZlPmcv.

21. María Victoria Cisneros, "Relaciones de desigualdad y seguridad social en países de baja cobertura. Caso de estudio: Ecuador, período 1923-2015" (tesis doctoral, FLACSO Ecuador, 2018), 102, https://bit.ly/3eHtRaZ.

22. Luis Francisco Sánchez Cáceres, "El sistema de Hard-Law y Soft-Law en relación con la defensa de los derechos fundamentales, la igualdad y no discriminación", Cuadernos Electrónicos de Filosofía del Derecho, . $^{\circ} 39$ (2019): 467-88.

23. ONU, Asamblea General, Declaración Universal de Derechos Humanos, 10 de diciembre de 1948, núm. 217 A (III), arts. 22 y 25. 
instrumentos garantizan el derecho a la seguridad social como derecho universal inalienable, con base en el principio de igualdad y no discriminación, ${ }^{24}$ recordando que la obligación asumida por los Estados parte del PIDESC es un instrumento de hard law. ${ }^{25}$

En el contexto de la OIT, Ecuador ha asumido obligaciones en virtud de los distintos convenios con esta organización como es el Convenio 102 de la OIT; en 2012 la OIT emitió la Recomendación 202 sobre los pisos de protección social. ${ }^{26}$ En este instrumento se crean los pisos nacionales de protección social, que reconocen o garantizan un mínimo relacionado a la salud esencial y la seguridad básica. Beccaria y Maurizio consideran que el principio de universalidad en la seguridad social no significa la búsqueda de los niveles mínimos de satisfacción de los derechos económicos y sociales básicos, sino el avance hacia un alto grado de homogeneidad. ${ }^{27}$

En el marco regional americano la Convención Americana reconoce el principio de igualdad y no discriminación mediante el cual todas las personas son iguales ante la ley, y es compromiso de los Estados parte el adoptar providencias tanto a nivel interno como en el marco de cooperación internacional, especialmente económica y técnica, para lograr la plena efectividad de los derechos que se derivan de las normas económicas, sociales y sobre educación, ciencia y cultura, contenidas en la Carta de la Organización de los Estados Americanos. El Protocolo Adicional a la Convención Americana sobre Derechos Económicos, Sociales y Culturales, Protocolo San Salvador, determina que "toda persona tiene derecho a la seguridad social que la proteja contra las consecuencias de la vejez y de la incapacidad que la imposibilite física o mentalmente para obtener los medios para llevar una vida digna y decorosa". ${ }^{28} \mathrm{La}$ Carta Internacional Americana de Garantías Sociales o Declaración de los Derechos Sociales del Trabajador: en el art. 2 señala el compromiso de los Estados parte en garantizar medidas de previsión y seguridad social.

24. Román Navarro Fallas, "El derecho fundamental a la seguridad social, papel del Estado y principios que informan la política estatal en seguridad social", Revista de Ciencias Administrativas y Financieras de la Seguridad Social 10, n. ${ }^{\circ} 1$ (2002): 13-8, https://bit.ly/3964Tks.

25. ONU, Asamblea General, Pacto Internacional de Derechos Económicos, Sociales y Culturales, 16 de diciembre de 1966, núm. 2200 A (XXI), art. 2.

26. OIT, R202 Recomendación sobre los pisos de protección social, 30 de mayo de 2012, núm. 202, https:// bit.ly/39bafLb.

27. Luis Beccaria y Roxana Maurizio, "Hacia la protección social universal en América Latina: una contribución al debate actual". Revista Problemas del Desarrollo 45, n. ${ }^{\circ} 177$ (2014): 37-58, https://bit.ly/35hu0AD.

28. Corte IDH, "Sentencia del 28 de febrero de 2003 (Excepciones Preliminares, Fondo, Reparaciones y Costas)", Caso "Cinco Pensionistas" vs Perú, 28 de febrero de 2003, párrs. 164-5. 
En el marco del acceso universal en materia de derechos sociales la Corte IDH considera que el acceso universal basado en los principios derechos humanos requiere que todos los bienes, servicios e información estén disponibles y sean asequibles para todos. ${ }^{29}$

\section{ANÁLISIS DEL SISTEMA DE SEGURIDAD SOCIAL CONTRIBUTIVO Y NO CONTRIBUTIVO EN ECUADOR}

La seguridad social se ha desarrollado con base en los modelos de Bismark y Bevedrige ${ }^{30}$ se considera que en Ecuador el sistema de pensiones nació bajo el modelo de Bismark, pero no se desarrolló industrialmente como en Europa. ${ }^{31}$ Con el modelo bismarkiano, surge en este país el esquema contributivo legal bilateral, en el cual el afiliado a cambio de una contribución recibe una prestación, en el que la cobertura depende de la condición laboral del individuo. ${ }^{32}$

La doctrina ha realizado una clasificación histórica de patrones de desarrollo entre países pioneros, intermedios y tardíos en la protección social y Ecuador se encuentra entre uno de los pioneros del grupo intermedio. ${ }^{33}$ De esta forma a través de la creación de la Caja de Pensiones, mediante el Decreto Ejecutivo n. ${ }^{\circ} 18$ del año 1928, inicia el modelo de seguros sociales en Ecuador ${ }^{34} \mathrm{y}$ en el entramado constitucional a partir de la Constitución de 1929. ${ }^{35}$ La seguridad social ha ido desarrollándose paulatinamente, mediante el reconocimiento como derecho irrenunciable de los trabajadores que se extiende a sus familiares, ${ }^{36}$ en el que se reconoce la autonomía de la institución que presta el servicio; ${ }^{37}$ a partir de la Constitución de 1979, se busca la extensión de la

29. Corte IDH, Cuadernillo de Jurisprudencia de la Corte Interamericana de Derechos Humanos $n .^{\circ} 22$ : Derechos Económicos, Sociales, Culturales y Ambientales (Costa Rica), párr. 113, https://bit.ly/3hoO06J.

30. Alfredo Sánchez Castañeda, "Principales modelos de seguridad social y protección social", La seguridad social y la protección social en México: su necesaria reorganización, n. ${ }^{\circ} 2$ (2012): 5-23, https://bit. ly/3ijRQ2M.

31. María Victoria Cisneros Campaña, "Sistema de pensiones en el Ecuador", Desigualdades, exclusión y crisis de sustentabilidad en los sistemas previsionales de América Latina y el Caribe (2018): 233-58, https:// bit.ly/2VAPe6X.

32. Angélica Porras Velasco, "La seguridad social en Ecuador: un necesario cambio de paradigmas", FORO: Revista de Derecho, n. ${ }^{\circ} 24$ (2015): 89-116, https://bit.ly/2BYJ2yz.

33. OIT, Panorama temático laboral. Presente y futuro de la protección social, 13-6.

34. Porras Velasco, "La seguridad social en Ecuador", 99.

35. Ecuador, Constitución de la República del Ecuador, Registro Oficial 138, 26 de marzo de 1929, art. 151, núm. 18.

36. Es importante destacar que, en la Constitución de 1945, la asistencia social se considera un servicio público que debe ser prestado por el Estado, de manera ineludible. Ecuador, Constitución de la República del Ecuador, Registro Oficial 228, 6 de marzo de 1945, art. 149.

37. Ecuador, Constitución de la República del Ecuador, Registro Oficial 133, 25 de mayo de 1967, art. 66. 
seguridad social a toda la población, como también el financiamiento tripartito. ${ }^{38}$ En la Constitución de 1998, la seguridad social tiene un mayor desarrollo, ya sea como un deber primordial del Estado que busca extender de manera progresiva el Sistema Nacional de Seguridad Social a toda la población urbana y rural, con independencia de su condición laboral; además, delega al Instituto Ecuatoriano de Seguridad Social (IESS) la prestación del Seguro Social General Obligatorio. ${ }^{39}$

El derecho a la seguridad social tiene igual importancia que otros derechos; los avances de la Constitución de 1998 y de la vigente Constitución eliminaron la categorización de derechos y estableció la igualdad jerárquica de derechos constitucionales. ${ }^{40}$

En el caso de la Constitución de 2008 se observa el camino en la búsqueda de la universalización efectiva de la Seguridad Social, como es el caso de la prestación entregada a quienes no realicen trabajo no remunerado en el hogar y la extensión a toda la población urbana y rural del Seguro Universal Obligatorio.

La Corte Constitucional ecuatoriana reconoció el derecho a la seguridad social contemplado en el artículo 34 de la Constitución como un derecho irrenunciable de todas las personas y un deber del Estado; además determina que el Instituto Ecuatoriano de Seguridad Social es el responsable de la prestación de las contingencias del Seguro Universal Obligatorio a sus afiliados. ${ }^{41}$

El sistema de seguridad social ecuatoriano está formado por el Régimen del Seguro Social General Obligatorio, Régimen Voluntario y el Seguro Social Campesino; así como también por dos regímenes especiales: el de las Fuerzas Armadas y el de la Policía Nacional (no analizados) aplicables a las personas del área urbana y rural que laboren o no. Comprende también una parte no contributiva que se relaciona con las prestaciones que entrega el IESS, en efectivo o en especie a los afiliados e hijos, para garantizar la falta de ingresos laborales por enfermedad, discapacidad, maternidad, accidentes de trabajo, desempleo, vejez o muerte de un miembro de la familia; asistencia médica y apoyo familiar insuficiente. ${ }^{42}$

38. Ecuador, Constitución de la República del Ecuador, Registro Oficial 800, 27 de marzo de 1979, art. 29, num. 1.

39. Ecuador, Constitución de la República del Ecuador, Registro Oficial 1, 11 de agosto de 1998, arts. 58-61.

40. Según el art. 11 de la Constitución Política del Ecuador, todos los principios y los derechos son inalienables, irrenunciables, indivisibles, interdependientes y de igual jerarquía; Ecuador, Constitución de la República del Ecuador, Registro Oficial 449, 20 de octubre de 2008, art. 11, citado en Patricio Pazmiño Freire, "El estado de las cosas inconstitucionales en la seguridad jurídica", OISS, accedido 17 de julio de 2020, 4, https://bit.ly/2OBCmK2.

41. Ecuador, Corte Constitucional, "Sentencia", en Juicio n. ${ }^{\circ}$ 287-16-CC; Ecuador, Corte Constitucional, "Sentencia", en Juicio n. ${ }^{\circ}$ 287-16-SEP-CC, 31 de agosto de 2016.

42. OIT, Informe VI: Seguridad Social para justicia social y una globalización equitativa, 8 . 
De acuerdo con la Ley de Seguridad Social en Ecuador forman parte del Seguro Social General Obligatorio los trabajadores en relación de dependencia y autónomos; profesionales en libre ejercicio; dueños de un negocio o una empresa unipersonal; menores de edad independizados de sus padres; personas que realizan quehaceres domésticos; y demás que deseen afiliarse a este sistema en virtud de leyes o decretos especiales; en tanto que pertenecen al Régimen Voluntario aquellas personas que independientemente de su situación laboral aportan voluntariamente al IESS para beneficiarse de los mismos servicios y prestaciones que hace uso el afiliado al Seguro Social General Obligatorio; medida que aplica para ecuatorianos residentes en el exterior y para ecuatorianos y extranjeros que viven dentro del Ecuador. Por otro lado, pueden afiliarse al Seguro Social Campesino, aquellas personas que se dedican a la pesca artesanal, así como aquellos que laboran en campos rurales, en su favor o el de la comunidad, sin ningún tipo de remuneración por parte de un ente público o privado y que no tengan trabajadores que dependan de él. ${ }^{43}$

El Instituto Ecuatoriano de Seguridad Social (IESS) como institución pública con carácter nacional es el instrumento por medio del cual el sistema de seguridad social ecuatoriano se hace efectivo en Ecuador. Tiene su propia reglamentación y patrimonio, goza de personería jurídica y jurisdicción coactiva, su gestión de recursos es autónoma y se sustenta de aportes personales y patronales de los afiliados al IESS, fondos de reserva, descuentos, multas, intereses, utilidades de inversiones, de la contribución económica obligatoria del Estado (40\%), y demás rubros señalados en la Ley de Seguridad Social. ${ }^{44}$

Cabe recalcar que la contribución entregada por empleadores y trabajadores no grava impuestos; en su lugar, es deducible del impuesto a la renta ${ }^{45}$ para las personas que trabajan bajo relación de dependencia, así como para las empresas o patronos quienes deducen lo aportado al IESS y los sueldos como gastos deducibles en su contabilidad, del total de los ingresos ordinarios y extraordinarios gravados pertenecientes al período fiscal inmediatamente anterior. ${ }^{46}$

43. Ecuador, Ley de Seguridad Social, Registro Oficial 465, Suplemento, 30 de noviembre de 2001, art. 2.

44. Ibíd., art. 16-9.

45. Ecuador, Ley de Régimen Tributario Interno, Registro Oficial 463, Suplemento, 17 de noviembre de 2004, art. 36-7.

46. La tarifa del impuesto a la renta de personas naturales y sucesiones indivisas actualmente es de 0 a $35 \%$ dependiendo el nivel de ingresos del contribuyente; en tanto que, para las sociedades constituidas en el Ecuador, así como para las sucursales de sociedades extranjeras domiciliadas en el país y los establecimientos permanentes de sociedades extranjeras no domiciliadas en el país, aplica la tarifa del $25 \%$ sobre su base imponible. 
El aporte de los afiliados al IESS para recibir pensiones estatales al jubilarse es del $8,86 \%$; en tanto que, para seguros de salud y riesgos del trabajo, el aporte corresponde al $11,74 \%$, rubros que sumados alcanzan el $20,6 \%$ y que son financiados por el trabajador $(11,15 \%)$ y el empleador $(9,45 \%){ }^{47}$ Por su parte, el Estado contribuye con el $40 \%$ al IESS, derivado del Presupuesto General del Estado; lamentablemente, como el gobierno ecuatoriano es deudor del IESS por años, los empleadores y trabajadores son el sostén principal de la institución. Debería haber un trato igualitario al patrono, trabajador y gobierno, ya que, cuando el aporte social no es pagado por parte del patrono o trabajador, el IESS acciona el proceso coactivo, lo que no se aplica para el Estado cuya falta de colaboración desencadenó una crisis económica en esta entidad que afecta a la ciudadanía.

La contribución al IESS generada por la relación laboral tiene una naturaleza de carácter tributario, es impuesta por el Gobierno y exigida coactivamente; a pesar de ello, no se considera un tributo debido a que la recaudación de esta no forma parte del arca fiscal que satisface el gasto público; en efecto, a este tipo de aportación se le denomina contribución parafiscal. Héctor Villegas agrega que es una obligación recabada por entes públicos competentes para asegurar el financiamiento autónomo y garantizar un beneficio sociali, ${ }^{48}$ es decir, la parafiscalidad se fundamenta en el modelo asistencial ${ }^{49}$ y se lleva a cabo a través de contribuciones para lograr un bienestar social cuando el afiliado lo solicita, o tiene necesidad de él. ${ }^{50}$ Según lo planteado por Corey Keyes, el bienestar social abarca cinco dimensiones: integración social, aceptación social, contribución social, actualización social y coherencia social, ${ }^{51}$ y se remite según Gerald Cohen a la noción de justicia distributiva, en la que todas las personas tienen algo por igual cantidad y es una política que combate la pobreza. ${ }^{52} \mathrm{La}$ justicia distributiva se refleja cuando la administración gubernamental con el aporte tripartito invierte en instalaciones, planes de salud, atención social y proyectos o programas ejecutados por el poder estatal. ${ }^{53}$

47. Patricia González, "Elevar 4,7 puntos en aporte de los afiliados al IESS cubriría el déficit", El Comercio, 17 de abril de 2019, 3, https://bit.ly/38eS5Ym.

48. Milagros Villasmil Molero et al., "Contribuciones parafiscales: Factor determinante para la seguridad social", Revista de Ciencias Sociales (Ve) XXIV, n. ${ }^{\circ} 3$ (2018): introducción, https://bit.ly/2BibawZ.

49. Tratamiento individualizado o personalizado, a una persona o grupo de personas.

50. Villasmil Molero et al., "Contribuciones parafiscales: Factor determinante para la seguridad social”, introducción, https://bit.ly/2BibawZ.

51. Corey Keyes, Social well-being: Social Psychology Quarterly (1998), 61, citado en Amalio Blanco y Darío Díaz, "El bienestar social: su concepto y medición", Revista Psicothema 17, n. 4 (2005): 582, https:// bit.ly/3hcmoSr.

52. Gerald Cohen, Igualdad de qué: sobre el bienestar, los bienes y las capacidades (Ciudad de México: CIECAS / IPN, 2004), citado en Rubén Oliver Espinoza, "Inclusión y exclusión: política social y de estabilización para el bienestar”, Mundo Siglo XX1, n. ${ }^{\circ} 15$ (2009): 88, https://bit.ly/33fZvbS.

53. Villasmil Molero et al., "Contribuciones parafiscales: Factor determinante para la seguridad social", introducción. 
Los aportes personales y patronales al IESS son parte del sistema de seguridad social contributivo y manifiestan la realidad financiera y económica de las personas físicas o naturales al ser su indicador principal la renta, capital o patrimonio que posee ${ }^{54}$ característica que determina la capacidad contributiva o de pago del aportante sobre la tasa del IESS la cual es progresiva. ${ }^{55}$ Cabe mencionar que el hecho generador de la contribución al IESS constituye la remuneración que recibe el empleado por parte del patrono en relación de dependencia y el ingreso que percibe el profesional o persona autónoma fruto de su trabajo. Por otro lado, el sistema de seguridad social no contributivo formado por prestaciones de dinero protege integralmente a los habitantes a través de atención médica y otros beneficios que satisfacen las necesidades de la población económicamente activa y retirada (jubilados) o en estado de vulnerabilidad (viudez, invalidez). ${ }^{56}$

Tabla 1. Sistema de seguridad social contributivo: aportaciones patronales y del trabajador

\begin{tabular}{|l|l|l|}
\hline $\begin{array}{c}\text { Afiliados al } \\
\text { Seguro Social } \\
\text { General } \\
\text { Obligatorio }\end{array}$ & $\begin{array}{c}\text { Afiliados } \\
\text { al Régimen } \\
\text { Voluntario }\end{array}$ & \multicolumn{1}{|c|}{ Afiliados al Seguro Social Campesino } \\
\hline $\begin{array}{l}\text { Enfermedad. } \\
\text { Maternidad. } \\
\begin{array}{l}\text { Riesgos del } \\
\text { trabajo. }\end{array}\end{array}$ & $\begin{array}{l}\text { Enfermedad. } \\
\text { Maternidad. } \\
\text { Riesgos del } \\
\text { trabajo. }\end{array}$ & $\begin{array}{l}\text { Enfermedades no profesionales de primer nivel de complejidad } \\
\text { médica y los servicios médico-asistenciales de mayor com- } \\
\text { plejidad; recuperación y rehabilitación de la salud del in- } \\
\text { dividuo; atención odontológica; y atención del embarazo, } \\
\text { parto y puerperio; excepto contingencia de enfermedad } \\
\text { al campesino jubilado. Ofrece programas de saneamiento } \\
\text { ambiental y desarrollo comunitario de las áreas rurales. }\end{array}$ \\
\hline
\end{tabular}

Fuente: Ley de Seguridad Social.

Elaboración propia.

Los tres regímenes sociales analizados aportan mensualmente al IESS para recibir atención de calidad, igualitaria y universal por parte del Estado; sin embargo, existe diferenciación en servicios de cobertura que son ineficientes y tardíos, la tecnología es antigua o inexistente y el personal no se abastece con la cantidad de pacientes que

54. Sin considerar las sociedades con o sin personería jurídica.

55. Ceferino Albano Costa González, "Los principios de equidad, capacidad contributiva e igualdad. Breve síntesis conceptual", Centro Interamericano de Administraciones Tributarias CIAT, 21 de marzo de 2017 , párr. 7-14, https://bit.ly/3dMWUtb.

56. Villasmil Molero et al., "Contribuciones parafiscales: Factor determinante para la seguridad social", resumen. 
reciben a diario; la medicación es de baja calidad, escasa o no hay; por lo que, según este panorama, no se cumple la universalidad efectiva en Ecuador.

\section{Tabla 2. Sistema de seguridad social no contributivo: prestaciones estatales}

\begin{tabular}{|c|c|c|}
\hline $\begin{array}{l}\text { Afiliados al Seguro Social } \\
\text { General Obligatorio }\end{array}$ & $\begin{array}{c}\text { Afiliados al Régimen } \\
\text { Voluntario }\end{array}$ & $\begin{array}{c}\text { Afiliados al Seguro Social } \\
\text { Campesino }\end{array}$ \\
\hline Jubilación ordinaria de vejez. & Jubilación ordinaria de vejez. & Invalidez total y permanente. \\
\hline $\begin{array}{l}\text { Jubilación por invalidez que } \\
\text { incluye discapacidad. }\end{array}$ & $\begin{array}{l}\text { Jubilación por invalidez que } \\
\text { incluye discapacidad. }\end{array}$ & Vejez. \\
\hline Jubilación por edad avanzada. & Jubilación por edad avanzada. & Auxilio para funerales. \\
\hline $\begin{array}{l}\text { Subsidio transitorio por } \\
\text { incapacidad. }\end{array}$ & $\begin{array}{l}\text { Subsidio transitorio por } \\
\text { incapacidad. }\end{array}$ & Discapacidad. \\
\hline $\begin{array}{l}\text { Trabajo del hogar no } \\
\text { remunerado. }\end{array}$ & $\begin{array}{l}\text { Trabajo del hogar no } \\
\text { remunerado. }\end{array}$ & Viudez. \\
\hline Incapacidad permanente total. & Incapacidad permanente total. & Orfandad. \\
\hline $\begin{array}{l}\text { Incapacidad permanente } \\
\text { absoluta. }\end{array}$ & $\begin{array}{l}\text { Incapacidad permanente } \\
\text { absoluta. }\end{array}$ & 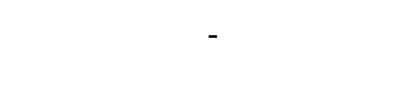 \\
\hline Montepío por viudez. & Montepío por viudez. & - \\
\hline Montepío por orfandad. & Montepío por orfandad. & - \\
\hline Subsidio para funerales. & Subsidio para funerales. & - \\
\hline $\begin{array}{l}\text { Rentas permanentes parciales } \\
\text { de riesgos del trabajo y del } \\
\text { seguro general. }\end{array}$ & $\begin{array}{l}\text { Rentas permanentes parciales } \\
\text { de riesgos del trabajo y del } \\
\text { seguro general. }\end{array}$ & - \\
\hline $\begin{array}{l}\text { Jubilados y beneficiarios de } \\
\text { montepío de los Ferrocarriles } \\
\text { del Estado. }\end{array}$ & $\begin{array}{l}\text { Jubilados y beneficiarios de } \\
\text { montepío de los Ferrocarriles } \\
\text { del Estado. }\end{array}$ & - \\
\hline Decimotercera pensión. & Decimotercera pensión. & - \\
\hline Decimocuarta pensión. & Decimocuarta pensión. & - \\
\hline Fondos de reserva. & Fondos de reserva. & - \\
\hline Cesantía. & Cesantía. & - \\
\hline Seguro de desempleo. & Seguro de desempleo. & - \\
\hline
\end{tabular}

Fuente: Ley de Seguridad Social.

Elaboración propia.

Los afiliados al Seguro Social General Obligatorio y al Régimen Voluntario aportan económicamente al IESS más que el Seguro Social Campesino porque reciben remuneraciones más altas; sin embargo, los pertenecientes al Seguro Social Campesino con necesidades más contundentes no tienen acceso a las mismas prestaciones, brecha 
social que debiera cubrir el Estado ecuatoriano para garantizar la universalidad del sistema de seguridad social.

En cuanto al desempleo, según Adil Mouhammed, el economista británico John Maynard Keynes (5 de junio de 1883-21 de abril de 1946) expresó que el origen del desempleo es siempre involuntario, cíclico y está directamente relacionado con las expectativas de los empresarios acerca del futuro de la economía; cuando no son positivas, invierten menos y el empleo termina por ceder. ${ }^{57}$ Ante ello, Andrea Toledo sugiere un modelo de seguro de desempleo aplicable en Ecuador cuando las condiciones de elegibilidad consideran, según Walter Nicholson y Karen Needels, tres requisitos: monetarios, que consisten en haber tenido empleo en un período determinado de tiempo; no monetarios, referentes a que el desempleo es involuntario y se evidencie una búsqueda activa de empleo por parte del asegurado; $\mathrm{y}$, finalmente, de continuidad, que radica en la persistencia de las condiciones anteriores para recibir el beneficio. ${ }^{58}$

Conforme se incrementan las prestaciones por desempleo, disminuye la búsqueda de trabajo. Sin embargo, la autora resalta que esta relación inversa caracteriza a los regímenes de prestaciones asistenciales que no exigen requisitos de acceso a la prestación y entregan beneficios ilimitadamente; en tanto que la prestación por desempleo no la reciben todos los desempleados, existen varios filtros para su acceso. Estas características incentivan a que el beneficiario de la prestación no se instale en la condición de desempleo. ${ }^{59}$

En cuanto a trabajadores informales, el desafío es mayor para el Gobierno, porque involucra personas de tercera edad, adolescentes, personas de escasos recursos, entre otras, que perciben ingresos económicos mínimos y no tienen acceso a beneficios sociales. ${ }^{60}$

57. Adil Mouhammed, Important Theories of Unemployment and Public Policies (2011), 12, citado en Andrea Carolina Toledo Guerrero, "Un modelo de seguro de desempleo para el sistema de seguridad social en Ecuador" (tesis de maestría, FLACSO Ecuador, 2016), 14-5, https://bit.ly/2Fk5pAk.

58. Walter Nicholson y Karen Needels, Unemployment Insurance: Strengthening the Relationship between Theory and Policy (2006), citado en Toledo Guerrero, ibíd., 14-5, https://bit.ly/2Fk5pAk.

59. Ibíd., 17-9.

60. Beccaria y Maurizio, "Hacia la protección social universal en América Latina: una contribución al debate actual”, 41-3, https://bit.ly/35hu0AD. 


\section{PRINCIPIO DE UNIVERSALIDAD DESDE UNA PERSPECTIVA EFECTIVA EN EL CONTEXTO ECUATORIANO}

En cuanto a cobertura, uno de los últimos avances fue el reconocimiento y universalización del seguro social al trabajador no remunerado, cuya participación en el mercado laboral según el Instituto Nacional de Estadística y Censos (INEC) representa el $19,1 \%,{ }^{61}$ Ecuador fue el primero de América Latina; ${ }^{62}$ sin embargo, a nivel nacional se redujo el número de empleos (aportantes) y la inestabilidad laboral aumentó, trayendo como consecuencia un déficit de dinero para el IESS, debido a que los gastos públicos de la institución fueron mayores a los ingresos percibidos, especialmente en la salud; además, se intuye la existencia de una red de corrupción por parte de algunos funcionarios de la entidad, así como la aplicación de sobreprecios en la construcción de áreas necesarias para el IESS y en la adquisición de equipos e insumos médicos a los diferentes proveedores, profundizando más la situación. ${ }^{63}$

Rodrigo Ibarra, experto actuarial, mencionó en el año 2013 que el IESS requería una transformación integral, con medidas que permitan mejorar los ingresos y bajar los egresos del fondo; subrayó también que no se puede traspasar todo el peso de la crisis en pensiones, a trabajadores y jubilados donde el Estado también es parte. ${ }^{64}$ Por su parte, el Banco del Instituto Ecuatoriano de Seguridad Social (BIESS), entidad filial o controlada por el IESS, también atraviesa un déficit económico y la sostenibilidad a corto y largo plazo está en riesgo. Una solución es hacer una auditoría, detectar y sancionar a los verdaderos responsables de la crisis económica, política y social en la que se encuentran dichas instituciones, ya que queda la duda si el IESS y el BIESS nos amparan o nos desfalcan económicamente, en medio de una economía decreciente.

Daniel Lemus Sares indica que actualmente los afiliados, jubilados y pensionistas que desean endeudarse y pagar en menos de un año (corto plazo) con tasas de interés del 8 al $11 \%$, dependiendo del tiempo en el cual van a saldar su deuda, pueden obtener un crédito quirografario preferencial otorgado por el BIESS con la condición de que, si el pago se lo realiza en más de un año (largo plazo), se aplica las tasas de interés

61. Ecuador, Instituto Nacional de Estadística y Censos, "Cuentas satélite del trabajo no remunerado de los hogares", Instituto Nacional de Estadística y Censos, accedido 17 de julio de 2020, tabla, https://bit. ly/32yNpvz.

62. Ecuador, Ministerio de Inclusión Económica y Social, “Trabajadoras no remuneradas en el hogar dialogan sobre su derecho a recibir afiliación social”, Ministerio de Inclusión Económica y Social, accedido 23 de junio de 2020, párr. 3-5, https://bit.ly/38rG8il.

63. Francisco Verni, "La mora patronal con el IESS asciende a \$ 1.196,4 millones en Ecuador", El Universo, 6 de octubre de 2019, 21-4, https://bit.ly/2NKLcVu.

64. Ibíd., 21-4. 
vigentes para el crédito quirografario regular, ${ }^{65}$ beneficio que en realidad se convierte en un subsidio estatal y que tiene que ser compensado. Según Lemus, "aceptar el sistema de préstamos quirografarios a tasas de interés inferiores a las del mercado significa pues sacrificar consumo futuro por presente"; asimismo, propone que: "Si se van a entregar créditos subsidiados estos solo deben ir dirigidos hacia aquellas actividades que se quieren beneficiar. A las demás no se les debe entregar dichos créditos o en su defecto hacerlo sin subsidios y solo con el fin de mejorar el rendimiento de la cartera de inversión del Seguro Social". Por tanto, si se recupera el dinero de las carteras vencidas del IESS y del BIESS, y se invierte en estas instituciones, el sistema de seguridad social sería fortalecido económicamente y tendría una reserva para sobrellevar las necesidades presentes, pero sobre todo futuras. ${ }^{66}$

Desde que el Estado interfiere en el sistema de seguridad social con el fin de distribuir riqueza equitativamente entre la población, se visualiza que en realidad el Estado se aprovecha de su poder de imperio para el dinero recaudado destinarlo a actividades urgentes del país en lugar de actividades importantes para el país. ${ }^{67}$ Ante ello, el Estado debiera ser imparcial, transparente y respetuoso con el dinero de los aportantes, sin descuidar el control y regulación que le compete; además, las autoridades deben trascender el concepto de seguridad social y valorar al IESS como aquella institución que permite a la ciudadanía tener una buena calidad de vida. Una reestructuración del sistema de seguridad social ecuatoriano, similar al que existió en el año 1942 con la Ley de Seguridad Social Obligatoria en la presidencia de Carlos Arroyo del Río, ${ }^{68}$ secaracterizó porque el patrono debía remitir los aportes personal y patronal a las dos Cajas del Seguro Social (pública y privada); es decir, a administraciones gubernamentales paralelas manejadas y administradas por organismos separados a la estructura del Estado central. Es una idea revolucionaria que pudiera enderezar el sistema actual, evitar el desvío de fondos y dar protección social bajo el control del Estado. ${ }^{69}$

Ecuador se encuentra identificado en un nivel intermedio en el desarrollo de su sistema de protección social en la región latinoamericana, con base en el índice multidimensional de protección social reconocido en el país; cabe destacar que este índice

65. Guillermo Lizarzaburo, "Quirografario preferencial del BIESS: menor plazo y mejor tasa de interés", Expreso, 24 de enero de 2020, 1-4, https://bit.ly/2NJPfRM.

66. Daniel Lemus Sares, "Préstamos quirografarios en el IESS", Perspectivas Económicas (Centro de Investigaciones Económicas CIEC-ESPOL), n. ${ }^{\circ} 3$ (2007): 1-2, https://bit.ly/3gdr7mH.

67. Ruiz Moreno, "Retos y desafíos de la seguridad social latinoamericana: entre la realidad y la utopía", 1156, https://bit.ly/3m2u3Gp.

68. Segundo período de gobierno comprendido entre el 1 de septiembre de 1940 hasta el 29 de mayo de 1944.

69. Carlos Andrés Orellana Jimbo, "La naturaleza jurídica del aporte a la seguridad social del trabajador en relación de dependencia y del empleador" (tesis de maestría, Universidad Andina Simón Bolívar, Sede Ecuador, 2019), 21-2, https://bit.ly/2ZpLB5H. 
fue analizado usando nueve indicadores, entre los cuales se encuentran el principio de universalidad y el principio de solidaridad, junto con el gasto público. ${ }^{70}$ En el caso de Ecuador ha demostrado un incremento de 10 puntos porcentuales en los últimos años según la OIT. ${ }^{71}$

Angélica Porras considera que la protección social ha sido desordenada en el país, no ha respondido a una política pública, sino a dar respuesta a puntos específicos de la población en respuesta a los afectados por las políticas neoliberales; en consecuencia, no existe una respuesta al desempleo o la pobreza. ${ }^{72}$

\section{CONCLUSIONES}

La cobertura legal existente en Ecuador crea un límite al ejercicio del derecho a la seguridad social, ya que el legislador, mediante la extensión de cobertura dirigida a ciertos sectores de la población, excluye a otros, generando vacíos de protección social; lo cual impide que sea una herramienta de justicia social plena, de igualdad y lucha contra la erradicación de la pobreza, institucionalizando la exclusión del ejercicio de la seguridad social, por contener elementos diferenciadores en su acceso. Se requiere evaluar la funcionalidad y las falencias actuales del sistema de seguridad social para, luego de ello, plantear soluciones prácticas y viables que instauren un seguro social que cubra a todos los individuos dentro de una colectividad, sin considerar situación laboral, edad o género; donde la función del Estado sea la de colaborar con el $40 \%$, regular y controlar la entidad con una política pública adecuada que facilite el manejo integral y racional del IESS, del BIESS y del sistema en sí. Es urgente desarticular la corrupción y transparentar la gestión y administración del IESS y del BIESS, al mismo tiempo que el Estado garantice la seguridad jurídica para que actúe el poder judicial y se aplique medidas de control y sanción, con el objetivo de incentivar la inversión privada nacional y extranjera, crear empleos y progreso económico al Ecuador. Si bien en Ecuador el sistema de seguridad social está atado al empleo formal, conviene implementar mecanismos contributivos, no contributivos y recursos o medidas fiscales para reducir la desigualdad existente por nivel de ingresos y ampliar la cobertura efectiva a las personas desempleadas, trabajadores informales y a quienes no hayan realizado ningún aporte al IESS, ya que estas se encuentran en un constante

70. José Antonio Ocampo y Natalie Gómez, "Social Protection Systems in Latin America: An Assesment", Working Paper, n. 54 (2014), 14-76, citado en Josette Altmann Borbón, América Latina: Frente a las cambiantes condiciones de su desarrollo (San José: SEGIB / FLACSO, 2017): 108.

71. OIT, Panorama temático laboral. Presente y futuro de la protección social en América, 82.

72. Porras Velasco, "La seguridad social en Ecuador", 108. 
estado de desprotección social. Además de ello, debe flexibilizarse cierta normativa laboral para aumentar contrataciones de personal y disminuir despidos.

\section{BIBLIOGRAFÍA}

Beccaria, Luis, y Roxana Maurizio. "Hacia la protección social universal en América Latina: Una contribución al debate actual”. Revista Problemas del Desarrollo 45, n. ${ }^{\circ} 177$ (2014): 37-58. https://bit.ly/35hu0AD.

Bertranou, Fabio. "Restrictions, problems and dilemmas of social protection in Latin America: Facing the challenges from ageing and income security". Well-being and Social Policy 1, n. ${ }^{\circ} 1$ (2005): 33-54.

Blanco, Amalio, y Darío Díaz. "El bienestar social: su concepto y medición”. Revista Psicothema 17, n. $^{\circ} 4$ (2005): 582-9. https://bit.ly/3hcmoSr.

Calvo León, Jorge Iván. "Principios de seguridad social”. Revista Jurídica de Seguridad Social, n. ${ }^{\circ} 8$ (1998): 36-40, https://bit.ly/3bIxg9u.

Cisneros Campaña, María Victoria. "Relaciones de desigualdad y seguridad social en países de baja cobertura. Caso de estudio: Ecuador, período 1923-2015". Tesis doctoral, FLACSO Ecuador, 2018. https://bit.ly/2Vx4C4e.

—. "Sistema de pensiones en el Ecuador". Desigualdades, exclusión y crisis de sustentabilidad en los sistemas previsionales de América Latina y el Caribe (2018): 233-58. https://bit. ly/2VAPe6X.

Corte IDH. Cuadernillo de Jurisprudencia de la Corte Interamericana de Derechos Humanos $n .^{\circ}$ 22: Derechos Económicos, Sociales, Culturales y Ambientales (Costa Rica), párr. 113. https://bit.ly/3hoO06J.

—. "Sentencia del 4 de julio de 2006 (Excepciones Preliminares, Fondo, Reparaciones y Costas)". Caso Ximenez López vs Brasil. 4 de julio de 2006.

—. "Sentencia del 22 de noviembre de 2007 (Excepciones Preliminares, Fondo, Reparaciones y Costas)". Caso Albán Cornejo y otros vs Ecuador. 22 de noviembre de 2007.

—. "Sentencia del 28 de febrero de 2003 (Excepciones Preliminares, Fondo, Reparaciones y Costas)". Caso "Cinco Pensionistas" vs Perú. 28 de febrero de 2003.

—. "Sentencia del 19 de mayo de 2013 (Excepciones Preliminares, Fondo, Reparaciones y Costas)". Caso Vera y otra vs Ecuador. 19 de mayo de 2013.

—. "Sentencia del 21 de mayo de 2013 (Excepciones Preliminares, Fondo, Reparaciones y Costas)". Caso Suárez Peralta vs Ecuador. 21 de mayo de 2013.

Costa González, Ceferino Albano. "Los principios de equidad, capacidad contributiva e igualdad. Breve síntesis conceptual”. Centro Interamericano de Administraciones Tributarias CIAT. 21 de marzo de 2017. https://bit.ly/3dMWUtb.

De Quiroz, Juan Bernaldo. “Concepto y fundamento”. El Seguro Social en Iberoamérica 44 (1945): 11-6. doi: 10.2307/j.ctvckq2zk.4. 
De Sousa Santos, Boaventura. Sociología jurídica crítica para un sentido común en el Derecho. Bogotá: Trotta, 2009.

Ecuador. Corte Constitucional. "Sentencia”. Juicio n. "287-16-SEP-CC. 31 de agosto de 2016.

Ecuador. Instituto Nacional de Estadística y Censos. "Cuentas satélite del trabajo no remunerado de los hogares". Instituto Nacional de Estadística y Censos. Accedido 17 de julio de 2020, tabla. https://bit.ly/32yNpvz.

Ecuador. Ministerio de Inclusión Económica y Social. "Trabajadoras no remuneradas en el hogar dialogan sobre su derecho a recibir afiliación social". Ministerio de Inclusión Económica y Social. Accedido 23 de junio de 2020. https://bit.ly/38rG8il.

Ecuador. Constitución de la República del Ecuador. Registro Oficial 138, 26 de marzo de 1929.

-. Constitución de la República del Ecuador. Registro Oficial 228, 6 de marzo de 1945.

-. Constitución de la República del Ecuador. Registro Oficial 133, 25 de mayo de 1967.

-. Constitución de la República del Ecuador. Registro Oficial 800, 27 de marzo de 1979.

-. Constitución de la República del Ecuador. Registro Oficial 1, 11 de agosto de 1998.

-. Constitución de la República del Ecuador. Registro Oficial 449, 20 de octubre de 2008.

—. Ley de Seguridad Social. Registro Oficial 465, Suplemento, 30 de noviembre de 2001.

-. Ley de Régimen Tributario Interno. Registro Oficial 463, Suplemento, 17 de noviembre de 2004.

Espinoza, Rubén Oliver. "Inclusión y exclusión: política social y de estabilización para el bienestar”. Mundo Siglo XX1, n. 15 (2009): 87-90. https://bit.ly/33fZvbS.

Etchichury, Horacio Javier. "Universalidad y derechos sociales: para una revisión constitucional de las políticas sociales en Argentina". Revista Estudios Socio-Jurídicos, n. ${ }^{\circ} 1$ (2019): 327-54. doi: http://dx.doi.org/10.12804/revistas.urosario.edu.co/sociojuridicos/a.6501.

González, Patricia. "Elevar 4,7 puntos en aporte de los afiliados al IESS cubriría el déficit". El Comercio. 17 de abril de 2019. https://bit.ly/38eS5Ym.

Ham, Roberto. "De la solidaridad intergeneracional a la privatización de las pensiones". Carta Demográfica sobre México. Ciudad de México: Demos, 1996.

Lemus Sares, Daniel. "Préstamos quirografarios en el IESS”. Perspectivas Económicas (Centro de Investigaciones Económicas CIEC-ESPOL), n. ${ }^{\circ} 13$ (2007): 1-2. https://bit.ly/3gdr7mH.

Lizarzaburo, Guillermo. "Quirografario preferencial del BIESS: menor plazo y mejor tasa de interés". Expreso. 24 de enero de 2020. https://bit.ly/2NJPfRM.

OIT. Informe VI: Seguridad Social para justicia social y una globalización equitativa. Ginebra: OIT, 2011.

- Panorama temático laboral. Presente y futuro de la protección social en América Latina y el Caribe. Ginebra: OIT, 2018.

ONU, CEPAL, OIT. "Encrucijadas en la seguridad social argentina: reformas, cobertura y desafíos para el sistema de pensiones" (Buenos Aires: ONU / CEPAL / OIT, 2011): 141, https://bit.ly/3bOmhLy. 
ONU. Asamblea General. Declaración Universal de Derechos Humanos. 10 de diciembre de 1948, núm. 217, A (III).

-. Asamblea General. Pacto Internacional de Derechos Económicos, Sociales y Culturales. 16 de diciembre de 1966, núm. 2200 A (XXI).

Orellana Jimbo, Carlos Andrés. "La naturaleza jurídica del aporte a la seguridad social del trabajador en relación de dependencia y del empleador". Tesis de maestría, Universidad Andina Simón Bolívar, Sede Ecuador, 2019. https://bit.ly/2ZpLB5H.

Organización Internacional del Trabajo. "Una recaída del empleo". La revista de la OIT, n. ${ }^{\circ} 73$ (2011): 8-16. https://bit.ly/2NHKoAm.

Pazmiño Freire, Patricio. "El estado de las cosas inconstitucionales en la seguridad jurídica". OISS. Accedido 17 de julio de 2020. https://bit.ly/2OBCmK2.

Porras Velasco, Angélica. "La seguridad social en Ecuador: un necesario cambio de paradigmas". FORO: Revista de Derecho, n. ${ }^{\circ} 4$ (2015): 89-116. https://bit.ly/2BYJ2yz.

Proaño Maya, Marco. Seguridad social y sociedad democrática. Quito: Editorial Americana, 2014.

Rey del Castillo, Javier. “Seguridad social y universalidad: ¿son compatibles?”. Revista de Administración Sanitaria Siglo XXI, n. ${ }^{\circ} 3$ (2004): 409-31. https://bit.ly/2ZlPmcv.

Ruiz Moreno, Ángel Guillermo. "Retos y desafíos de la seguridad social latinoamericana: Entre la realidad y la utopía”. Revista Latinoamericana de Derecho Social, n. ${ }^{\circ} 12$ (2011): 113-47. https://bit.ly/3m2u3Gp.

Sánchez Cáceres, Luis Francisco. "El sistema de Hard-Law y Soft-Law en relación con la defensa de los derechos fundamentales, la igualdad y no discriminación". Cuadernos Electrónicos de Filosofía del Derecho, n. ${ }^{\circ} 39$ (2019): 467-88.

Sánchez Castañeda, Alfredo. "Principales modelos de seguridad social y protección social". La seguridad social y la protección social en México: su necesaria reorganización, $\mathrm{n}{ }^{\circ} 23$ (2012): 5-23. https://bit.ly/3ijRQ2M.

Tinoco, Elizabeth. "El desafío de la seguridad social en América Latina”. Organización Internacional del Trabajo. 6 de febrero de 2015.

Toledo Guerrero, Andrea Carolina. "Un modelo de seguro de desempleo para el sistema de seguridad social en Ecuador” (tesis de maestría, FLACSO Ecuador, 2016), 14-5. https:// bit.ly/2Fk5pAk.

Verni, Francisco. "La mora patronal con el IESS asciende a \$ 1.196,4 millones en Ecuador". El Universo. 6 de octubre de 2019. https://bit.ly/2NKLcVu.

Villasmil Molero, Milagros, Lisandro José Alvarado Peña, Cecilia Cristina Socorro González y Nery Gamboa Caicedo. "Contribuciones parafiscales: Factor determinante para la seguridad social”. Revista de Ciencias Sociales (Ve) XXIV, n. 3 (2018): introducción. https:// bit.ly/2BibawZ. 